\title{
The Application of Information Literacy with Association Rules for a Safe Cyber Environment System
}

\author{
Chia-Chia Lin ${ }^{1, a}$, Hung-Yuan Huang ${ }^{2, b}$, Wen-Tan $\mathrm{Wu}^{3, \mathrm{c}}$ \\ ${ }^{1}$ No.117 Chian-Kuo Rd., Sec. 2, Ming-Hsiung, Chia-Yi, 62153, Taiwan.(WuFeng University) \\ ${ }^{2} 2 F$., No. 38, Aly. 5, Ln. 100, Jiangcuo Rd., Douliou, Yunlin, 64002, Taiwan \\ ${ }^{3}$ No.13,Ln.59,Huian St., Ming-Hsiung, Chia-Yi, 62154,Taiwan(ToKo University) \\ a46cclin@gmail.com, bcash6263@yahoo.com.tw , 'cq2218470@gmail.com
}

Keywords: Information literacy, Data mining, Cyber Environment System

Abstract:The Internet is filled with opportunities for learning, communicating, and sharing information. It is a valuable resource for children and adults alike. Like any large community, however, the online world presents some underlying risks, especially for children. Parents need to be aware of some of the potential problems their children could encounter, and try to take adequate measures to protect their children from injury. In this paper, researcher can try to explore the relationship between parent's information literary, the confidence in child's ability of self-defense on the internet, and adequate measures to promote child using the internet more effectively. Association rules, a kind of data mining strategies, will be the main tool to manipulate the dataset.

\section{Introduction}

Over the past few years, a fast growing number of households have added electronic games, home computers, and the Internet to other technologies - the telephone, radio, TV, and stereo system - that consume children's time. Surveys of parents suggested that they bought home computers and subscribed to Internet access to provide educational opportunities for their children, and to prepare them for the "information-age". Although they were increasingly concerned about the influence of the Web on their children and expressed disappointment over their children using the computer for activities such as playing games and browsing the Internet to download lyrics of popular songs and pictures of rock stars, they generally considered time wasted on the computer preferable to time wasted on TV, and even considered children without computers to be at a disadvantage. Nowadays, multimedia and interactive live video technologies innovate the argument of "information-age". While digital content become more and more complicated and diverged from normal educational purport or entertainment, parents don't regard home computer and Internet as only a toy of cheating time anymore. Now, some of these parents concern that how to regulate the rules to oversee children's behavior of Internet access and conduct them use these equipments correctly.

In this paper, research will address the different associations, according to the level of information technology and knowledge these parents reach, between children's behavior or activity in using the internet and parents manner. In other words, this research focuses on how the different levels of information literate parents affect the strategies of regulating the rule and the role they play.

\section{Literature review}

Information literacy. Nowadays, the manipulation of new operation system in computer and peripheral seem to become more complicated, graphic interface doesn't substantively decrease the perplexity of operation, it is actually information literacy that should be the concern. Information literacy is much more than knowing how to open a Web browser and type a search term into Google. Information literacy is the ability to recognize when information is needed and to locate, evaluate, and use that information effectively. Moreover, information literacy is not just a skill required in college; there is "a lifelong need for being informed and up-to-date"."Information literacy includes cognitive activities, such as acquiring, interpreting, and evaluating the quality of information. It is 
enabled by technical skills, such as using a computer to research, organize, analyze, and communicate. Information literacy also is increasingly important in the contemporary environment of rapid technological change and proliferating information resources. Because of the escalating complexity of this environment, individuals are faced with diverse, abundant information choices - in their academic studies, in the workplace, and in their personal lives. "Information literacy is a survival skill in the Information Age... Information literate people know now to find, evaluate, and use information effectively to solve a particular problem or make a decision - whether the information they select comes from a computer, a book, a government agency, a film, or any number of other possible resources". Information literacy forms the basis for lifelong learning. It is common to all disciplines, to all learning environments, and to all levels of education. It enables learners to master content and extend their investigations, become more self-directed, and assume greater control over their own learning.

Data mining. While addressing the relationships between parent's information literacy and children's attitude of using computer, some content of association between parents, children and computer may be worthy to explore more deeply. Data mining is one kind of tool often used to express the context of complicated structure. There are four basically different styles of learning appear in data mining application, they are classification learning, clustering, numeric predication, and association learning. In classification learning, the learning scheme is presented with a set of classified examples from which it is expected to learn a way of classifying unseen examples. In clustering, groups of examples that belong together are sought. In numeric predication, the outcome to be predicted is not a discrete class but a numeric quantity. In association learning, any association among features is sought, not just ones that predict a particular class value.

In this paper, association rule will be used to explore the dataset. Association rules have two special characteristics: they can "predict" any attribute, not just the class, and they can "predict" more than one attribute's value at a time.

\section{Data and methodology}

Data : UK Children Go Online Project (UKCGO). As part of a longer program of research on children and young people's uses of new media at the London School of Economics, two related projects have been designed and conducted, UK Children Go Online Project (UKCGO) is one of them. The UK Children Go Online Project (UKCGO) conducted between 2013 and 2015, this project surveyed 1631 children and young people aged 9-19 year olds across the UK in spring 2015, together with 967 parents of the 9-17 year olds. The survey asked a variety of detailed questions focused on young people's Internet access and use in order to ask how the Internet may be transforming, or may itself be shaped by, family life, peer networks and learning, formal and informal.

In this paper, parents' part of The UK Children Go Online Project (UKCGO) will be selected and deliberated on four subjects. First, whether or how parents understand the intention and content of children's manipulation on the internet? Second, how do parent keep with their children while they are on the internet? Third, is there any activity which is not permitted while children on the internet? The deviation of the strategies to there four subjects depends on the different level of information literacy which the parents have. The research of this paper will focus on exploring the relationship between information literacy and these four subjects.

Methodology: Apiori algorithm. Weka, a system developed at the University of Waikato in New Zealand, applies to this research, is a collection of machine learning algorithms for solving real-world data mining problems. It is written in Java and runs on almost any platform. The algorithms can either be applied directly to a dataset or called from your own Java code. Association rules, a kind of data mining strategies, will be the main tool to manipulate the dataset, Apriori, a classic algorithm for learning association rules, is designed to operate on databases containing transactions

As is common in association rule mining, given a set of itemsets (for instance, sets of retail transactions each listing individual items purchased), the algorithm attempts to find subsets which are common to at least a minimum number $\mathrm{C}$ (the cutoff, or confidence threshold) of the itemsets. Apriori 
uses a "bottom up" approach, where frequent subsets are extended one item at a time (a step known as candidate generation), and groups of candidates are tested against the data. The algorithm terminates when no further successful extensions are found.

Apriori uses breadth-first search or a generate-and-test type structure to count candidate item sets efficiently. It generates candidate item sets of length $k$ from item sets of length $k-1$. Then it prunes the candidates which have an infrequent sub pattern, the candidate set contains all frequent $k$-length item sets. After that, it scans the transaction database to determine frequent item sets among the candidates.

Apriori, while historically significant, suffers from a number of inefficiencies or trade-offs, which have spawned other algorithms. Candidate generation generates large numbers of subsets (the algorithm attempts to load up the candidate set with as many as possible before each scan). Bottom-up subset exploration (essentially a breadth-first traversal of the subset lattice) finds any maximal subset $\mathrm{S}$ only after all $2^{|S|}-1$ of its proper subsets.

\section{Result and discussion}

In this research, the information literate parents (967 instances) are divided into four levels, according to their self-determination on how deep and wide they comprehend the information technology and knowledge. These four levels are beginner, average, advanced, and don't know (indefinable). The proportion of these parents in these different four levels are beginner $22.03 \%$ (213 instances), average $39.30 \%$ (380 instance), advanced 10.96\% (106instances), and don’t know 27.71\% (268 instances).

Table I Attributes and details of dataset

\begin{tabular}{|c|c|c|}
\hline Attribute & Detail & Value \\
\hline \multicolumn{3}{|c|}{ pq211 pq215: Does parent understand enough about the internet to do any of these things } \\
\hline pq211 & know what kinds of things the child does on the internet & Yes/No \\
\hline pq212 & know how to access child's email account & Yes/No \\
\hline pq213 & know how to check which websites child has visited & Yes/No \\
\hline pq214 & help child use the internet safely & Yes/No \\
\hline pq215 & help child get the best out of the internet & Yes/No \\
\hline
\end{tabular}

pq221 pq224: Do parent do any of these nowadays?

pq221 keep an eye on what's on the screen while the child is online Yes/No

pq222 check the computer later, to see which sites the child visited Yes/No

pq223 check the message in the child's email account Yes/No

pq224 get annoyed with the child about his/her use of the internet Yes/No

pq231 pq238: Some thing child is not allowed to do on the internet.

pq231 child is not allowed to give out personal information

pq232 child is not allowed use email

pq233 child is not allowed use chat rooms

pq234 child is not allowed to use instant messaging Yes/No

pq235 child is not allowed to play game on the internet $\quad$ Yes/No

pq236 child is not allowed to download things (e.g. music, games) Yes/No

pq237 child is not allowed to buy anything online Yes/No

pq238 child is not allowed to fill out online forms or quizzes Yes/No

After analysing the rules of first question, it is found that the parents of beginner and "don't know" lack ability to check which website the child has visited, and also, they can not help child get the best 
out of the internet. But to the most parents belong to the group of average and advanced, they know what activities the child does on the internet, and how to check the websites the child has visited, so these parents have the ability to help child use the internet safety.

From the rules extracted from the second question, research illustrates that these parents of beginner and "don't know" seem not to (or lack the ability to ) oversee the content on the screen or check the message in child's email account. While parents of average and advanced will check the sites the child visited, keep an eye on what's content, and don't get annoyed with child's use of the internet.

To most of the parents in the group of beginner and "don't know", they will not permit child using email and playing games on the internet if the child is not allowed to us instant messaging. But to another two groups of parents average and advanced, using chat rooms is not permitted besides playing games on the internet if child is not allowed to use instant messaging.

\section{Conclusions}

Appropriate restriction and conduct can provide children a safe cyber environment to use home computer and Internet access, the variation of parents' information literacy will regulate different rules, but same group of information literate parents seem to have similar manner and cognition. By data mining technology, the class owned same associations between attributes can be sieved out and try to regularize the rules. But it is not enough, further research will be necessary to deeply explore the causes and influences of these associations.

\section{References}

[1] American Library Association, Presidential Committee on Information Literacy: Final Report (Washington,D.C.:ACRL, January10, (1989).

http://www.ala.org/ala/acrl/acrlpubs/whitepapers/presidential.htm>.

[2] American Library Association, Presidential Committee on Information Literacy.

[3] Ian H.Witten, Eibe Frank, "DATA MINING-Practical Machine Learning Tools and Techniques" $2^{\text {nd }}$ edition, Elsevier, (2005).

[4] Kaveri Subrahmanyama, Patricia Greenfieldb, Robert Krautc, Elisheva Grossb, " The impact of computer use on children's and adolescents' development", Applied Developmental Psychology 22, (2001), pp.7-30.

[5] Kraut, R., Scherlis, W., Mukhopadhyay, T., Manning, J., \& Kiesler, S. The HomeNet field trial of residential Internet services. Communications of the ACM, 39, (1996), pp. 55-63.

[6] Livingstore, S., \& Bober, M. "UK children go on line: listening to young people's experience" London School of Economics and Political Science, (2003).

[7] The Association of College and Research Libraries, A division of the American Library Association, " The Information Literacy Competency Standards for Higher Education", Endorsed by the American Association for Higher Education, October 1999. and the Council of Independent Colleges, February 2004. available at: http://www.ala.org/acrl/ilcomstan.html.

[8] Turow, J. "The Internet and the family: The view from the parents, the view from the press" (Report No. 27). Philadelphia, PA: Annenberg Public Policy Center of the University of Pennsylvania, May 4, (1999). 